\title{
Boundaries of the $\delta$ Scuti Instability Region
}

\author{
Alexei A. Pamyatnykh \\ Copernicus Astronomical Center, Bartycka 18, 00-716 Warsaw, Poland \\ Institute of Astronomy, Pyatnitskaya Str. 48, 109017 Moscow, Russia
}

With new data on stellar opacity and equation of state, we computed the theoretical blue edges of the classical instability strip in the vicinity of the main sequence. The results were compared with observational data on $\delta$ Scuti variables using various diagrams (HR, $\log g-\log T_{\text {eff }}, M_{V}-(b-y)_{0}$ and so on). An example of these diagrams is shown in Fig. 1. Detailed results can be found in Pamyatnykh (2000).

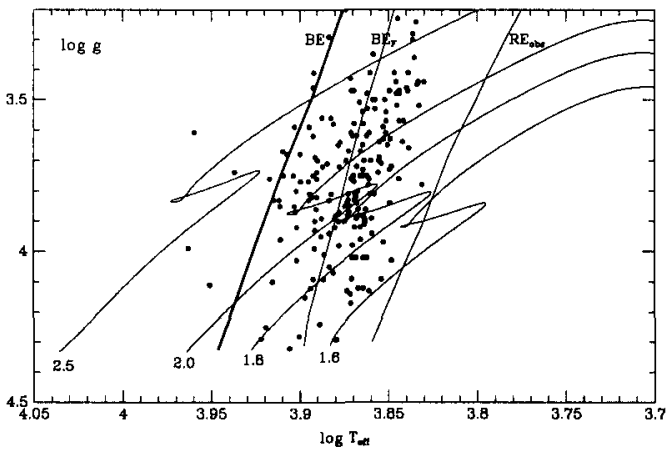

Figure 1. $\log g-\log T_{\text {eff }}$ diagram for $\delta$ Sct stars. The symbols BE, $\mathrm{BE}_{\mathrm{F}}$ and $\mathrm{RE}_{\mathrm{obs}}$ mark the theoretical blue edge for radial overtones, for the radial fundamental mode, and the empirical red edge, respectively. Evolutionary tracks of $1.6,1.8,2.0$ and $2.5 M_{\odot}$ models $(X=0.70$, $Z=0.02$ ) computed with OPAL opacities (Iglesias \& Rogers 1996) are shown. Observational data are due to Rodríguez et al. (1994).

Acknowledgments. The work was supported by the grants RFBR-98-0216734 and KBN-2-P03D-014-14.

\section{References}

Iglesias, C. A. \& Rogers, F. J. 1996, ApJ, 464, 943

Pamyatnykh, A. A. 2000, in ASP Conf. Ser., 6th Vienna Workshop in Astrophysics; Delta Scuti and Related Stars, ed. M. Breger \& M. Montgomery (San Francisco: ASP), in press

Rodríguez, E., Lopez de Coca, P., Rolland, A., Garrido, R., \& Costa, V. 1994, A\&AS, 106, 21 